\title{
PREDICTION OF DESIGN TYPHOON WIND SPEEDS AND PROFILES USING REFINED TYPHOON WIND FIELD MODEL
}

\author{
W.F. Huang ${ }^{1}$, Y.L. Xu ${ }^{2, *}$, C.W. $\mathrm{Li}^{3}$ and H.J. Liu ${ }^{4}$ \\ ${ }^{1}$ PhD Candidate, Shenzhen Graduate School, Harbin Institute of Technology, Shenzhen, China \\ ${ }^{2}$ Guest Professor, Shenzhen Graduate School, Harbin Institute of Technology, Shenzhen, China; Chair Professor, \\ Department of Civil and Structural Engineering, The Hong Kong Polytechnic University, Hong Kong \\ ${ }^{3}$ Professor, Department of Civil and Structural Engineering, The Hong Kong Polytechnic University, Hong Kong \\ ${ }^{4}$ Professor, Shenzhen Graduate School, Harbin Institute of Technology, Shenzhen, China \\ *(Corresponding author: E-mail: ceylxu@polyu.edu.hk)
}

Received: 20 May 2011; Revised: 24 July 2011; Accepted: 27 July 2011

\begin{abstract}
For buildings and structures in typhoon regions, they must be designed to withstand typhoon winds during their design lives. The determination of design typhoon wind speed for a structure within a given design life thus becomes an imperative task. A refined typhoon wind field model considering the influence of temperature and the variation of central pressure difference with height has been recently proposed by the authors. This paper aims to use this refined typhoon wind field model together with the Monte Carlo simulation and the typhoon wind decay model for predicting design typhoon wind speeds and profiles based on 60-year typhoon wind field data recorded by the Hong Kong Observatory. Both directional and non-directional design wind speeds of 50-year return period predicted by the refined typhoon wind field model, the Meng model and the Shapiro model for the Waglan Island of Hong Kong are compared with the statistical ones directly from the wind measurement data recorded by the anemometers installed on the Waglan Island. Averaged mean wind speed profiles at the Waglan Island predicted by the refined typhoon wind field model and the Meng model are also computed and compared with field measurement data available. The results show that the refined typhoon wind field model could predict design typhoon wind speeds and averaged wind profiles satisfactorily.
\end{abstract}

Keywords: Design wind speed, Averaged wind profile, Refined typhoon wind field model, Monte Carlo simulation, Typhoon wind data, Comparison

\section{INTRODUCTION}

Buildings and structures in typhoon regions are inevitably affected by typhoon winds. They must be designed to withstand typhoon winds during their design lives. The determination of design typhoon wind speed for a structure within a given design life thus becomes an imperative task. Although several approaches can be employed to estimate design typhoon wind speeds for buildings and structures, the use of the Monte Carlo simulation method in conjunction with a typhoon wind field model is a most universal approach.

The use of the Monte Carlo simulation method to determine design typhoon wind speeds for buildings and structures was probably first suggested by Russell [1,2]. Within the simulation, probability distributions of typhoon parameters, such as annual occurrence rate, paths, central pressure difference, translation velocity, approach angle, minimum of closest distance, radius to maximum winds, were first determined from the observation data. The typhoon wind field model with/without the typhoon decay model after landfall was then used to generate a series of typhoons by using the Monte Carlo simulation. The design wind speed for a given return period and location was finally decided through the extreme wind analysis. The method suggested by Russell was then expanded and improved by many researchers including Martin [3], Tryggvason et al. [4], Batts et al. [5], Georgiou et al. [6], Fujii and Mitsuta [7], Vickery and Twisdale [8], and Vickery et al. [9]. The study by Batts et al. [5] was a milestone, being the first study to examine the entire United States coastline, and it provided a rational means to estimate typhoon wind speeds along the Gulf and Atlantic coasts of the United States. Vickery and Twisdale [9] developed an improved prediction 
methodology for typhoon wind speeds with an emphasis placed on the importance of typhoon wind field model and decay model used in Vickery and Twisdale [8]. Vickery et al. [10] further developed the simulation approach where the typhoon full track and the central pressure varying with the sea surface temperature were modeled.

To determine design typhoon wind speeds using the Monte Carlo simulation, the modeling accuracy of typhoon wind field in the atmospheric boundary layer is a key part. The modeling of typhoon wind field has been investigated by many researchers, such as Chow [11], Gomes and Vickery [12], Holland [13], Batts et al. [5], Shapiro [14], Georgiou [15], Thompson and Cardone [16], Vickery and Twisdale [8], and Meng et al. [17] among others. All these existing models do consider the physical features of the typhoon boundary layer caused by friction at the earth's surface, but they often neglect the influence of temperature and assume that the central pressure difference of a typhoon does not vary with height above the ground. The numerical simulation results obtained by meteorologists suggested that it was necessary to include temperature due to dissipative heating from the surface friction in forecasting typhoon structure and intensity (Jin et al. [18]). The field observations made from the data recorded by the GPS dropsonde (Kepert [19]) showed that the pressure of a typhoon actually decreased with increasing height above the ground. The assumption that the central pressure difference remains constant with height may affect the prediction of design typhoon wind speed and averaged wind profile. A refined typhoon wind field model including the influence of temperature and the variation of central pressure difference with height was recently proposed by the authors based on the three dimensional Navier-Stokes equations (Huang and $\mathrm{Xu}[20]$ ). The wind speed and direction computed from the refined typhoon wind field model were compared with those measured at the Waglan Island and the Di Wang Tower, and those predicted by the Meng model and the Shapiro model. The spatial distribution of wind speed in a horizontal plane was also given through an isotach analysis and compared with the visual imagery of Typhoon York obtained by the Hong Kong Observatory (HKO). Mean wind speed profiles of Typhoon York at different distances from typhoon center were computed and examined through the comparison with field data and power-law profiles. The results demonstrated that the refined typhoon wind field model gave more accurate wind speed, direction and profile than other models.

The refined typhoon wind field model together with the typhoon wind decay model is used in this paper in conjunction with the Monte Carlo simulation method to predict design typhoon wind speeds and wind profiles in Hong Kong. After a brief description of the refined typhoon wind field model and the typhoon wind decay model, the possible statistical distributions of typhoon parameters are established based on typhoon wind data recorded by the HKO between 1947 and 2006 within a $250 \mathrm{~km}$ distance. The Kolmogorov-Smirnov $(K-S)$ test is then used to determine which statistical distribution can best fit the typhoon wind data for every typhoon parameter. Since there is a long-term observation station at the Waglan Island of Hong Kong, it is selected in this study to verify the refined typhoon wind field model in terms of design wind speeds. To this end, Monte Carlo simulation method is used to generate a series of typhoons for the Waglan Island in conjunction with the refined typhoon wind field model, the typhoon wind decay model, and the statistical distributions of typhoon parameters. For each typhoon, the hourly mean wind speed and direction at the anemometer height of the Waglan Island are obtained. The same approach, but using the Shapiro model and the Meng model instead of the refined typhoon wind field model, is also applied to generate a series of typhoons for the Waglan Island and to determine the hourly mean wind speed and direction from each typhoon at the anemometer height of the Waglan Island. On the other hand, the hourly mean wind speed and direction recorded by the anemometer due to typhoons at the Waglan Island from 1970 to 1999 are available. Based on the four sets of data, the non-directional and directional design wind speeds of 50-year return period at the Waglan Island anemometer height are analyzed and compared. Furthermore, mean wind speed profiles at the 
Waglan Island are also generated by the refined typhoon wind field model and the Meng model for a series of typhoons during the computation simulation. Averaged mean wind speed profiles from the refined typhoon wind field model and the Meng model are computed and compared with the field measurement data available.

\section{REFINED TYPHOON WIND FIELD MODEL}

The refined typhoon wind field model and the typhoon wind decay model, which are used in the Monte Carlo simulation for predicting typhoon design wind speeds and wind profiles, are briefly described in this section for the sake of completion. The detailed information can be found in Huang and $\mathrm{Xu}[20]$.

The deduction of the refined typhoon wind field model is based on the three dimensional Navier-Stokes equations (Holton [21]). To obtain the typhoon wind field for predicting design wind speeds for buildings and structures, the hydrostatic approximation is adopted in the refined typhoon wind field model because the vertical scale of a typhoon is much less than its horizontal scale. The air density is also assumed to be constant. Furthermore, to consider topographic effects, a geometric height based terrain-following coordinate transformation is used to deal with the difficulties at lower boundary that arise from using the conventional $z$-coordinate. Based on the above assumptions and after some manipulations, the Navier-Stokes equations become

$$
\begin{aligned}
& \frac{d \boldsymbol{v}_{h}}{d t}=-\frac{1}{\rho} \nabla_{h} p-f \boldsymbol{k}_{h} \times \boldsymbol{v}_{h}+\boldsymbol{F}_{h} \\
& p=p_{0}\left(1-g z / \theta c_{p}\right)^{c_{p} / R}
\end{aligned}
$$

where the subscript $h$ means the horizontal; $\boldsymbol{v}_{h}$ is the wind velocity in the horizontal plane; $\boldsymbol{k}_{\boldsymbol{h}}$ is the unit vector in horizontal plane; $\boldsymbol{F}_{h}$ is the friction force in the horizontal direction; $\nabla_{h}$ is the two-dimensional del operator; $p$ is the atmospheric pressure; $\rho$ is the air density; $f$ is the Coriolis parameter; $p_{0}$ is the pressure at zero-plane; $z$ is the height from ground surface; $\theta$ is the potential temperature; $c_{p}$ is the heat capacity at constant pressure; $g$ is the gravitational acceleration; and $R$ is the ideal gas constant.

Eqs. 1 and 2 cannot be directly solved because the number of unknown quantities is greater than the number of equations. Nevertheless, Holland [13] proposed the following analytical model for the radial profiles of sea level pressure in a typhoon based on field measurement data.

$$
p_{0}=p_{c 0}+\Delta p_{0} \exp \left[-\left(r_{m} / r\right)^{B}\right]
$$

where $p_{c 0}$ is the central pressure of a typhoon at zero-plane; $\Delta p_{0}$ is the central pressure difference at zero plane equal to $p_{m 0}-p_{c 0} ; p_{m 0}$ is the ambient pressure (theoretically at infinite radius) at zero-plane; $r_{m}$ is the radius to maximum winds; $r$ is the radial distance from the typhoon center; and $B$ is the Holland's radial pressure profile parameter, taking on values between 0.5 and 2.5. Eq. 1,2 and 3 are the basic equations of the refined model for typhoon wind field over sea. The basic equations can be solved by using a similar way as proposed by Meng et al. [17], in which typhoon-induced mean wind velocity is regarded as the combination of a friction-free wind and a surface wind caused by friction due to ground surface. The perturbation analysis is performed to find the solution. 
Once the typhoon makes landfall, it weakens as the central pressure rises. Proper modeling of the decay of the typhoon is important for the prediction of typhoon winds after landfall. The form of the decay model used in this paper is similar to one proposed by Vickery and Twisdale [8] for North America but the parameters are different. The decay model is defined as:

$\Delta p_{0}(t)=\Delta p_{0 n} \exp (-a t+b)$

Where $\Delta p_{0}(t)$ is the central pressure difference at zero plane of a typhoon at $t$ hours after landfall; $\Delta p_{0 n}$ is the central pressure difference at zero plane at the time when the typhoon makes landfall; $a$ is the decay constant; and $b$ is the random variable with a normal distribution. The decay constant $a$ is given as:

$a=a_{0}+a_{1} \Delta p_{0 n}+\varepsilon$

where $a_{0}$ and $a_{1}$ are the constants determined by a standard linear regression analysis; $\varepsilon$ is the error term with a zero-mean normal distribution. The basic equations of the refined model for typhoon wind field over sea (Eqs. 1-3) together with the typhoon wind decay model (Eqs. 4-5) can be used to predict the typhoon wind field over land.

\section{MONTE CARLO SIMULATION}

The design wind speed for a given return period can be determined by using the observation data of annual maximum wind speeds recorded at the concerned location. However, the observation data are often not enough for a reliable estimation of long return period design wind speed. For example, the annual maximum wind speeds of at least several thousand years are required to estimate the design wind speed with a return period of 50 years. Typhoon simulation is an alternative approach to evaluate extreme wind speeds over long periods of time by the so-called Monte Carlo simulation. To generate a series of typhoons using the Monte Carlo simulation method in conjunction with the typhoon wind field model and the typhoon wind decay model, the statistical distributions of six typhoon key parameters, i.e. the central pressure difference $\Delta p_{0}$, the translation velocity $c$, the approach angle $\theta$, the minimum of closest distance $d_{\min }$, the radius to maximum winds $r_{\mathrm{m}}$ and the annual occurrence rate $\lambda$, shall be established based on typhoon wind field data recorded in the area concerned. The area concerned in this study is around the Waglan Island in Hong Kong because of its location and the quality and quantity of wind data available (see Figure 1).

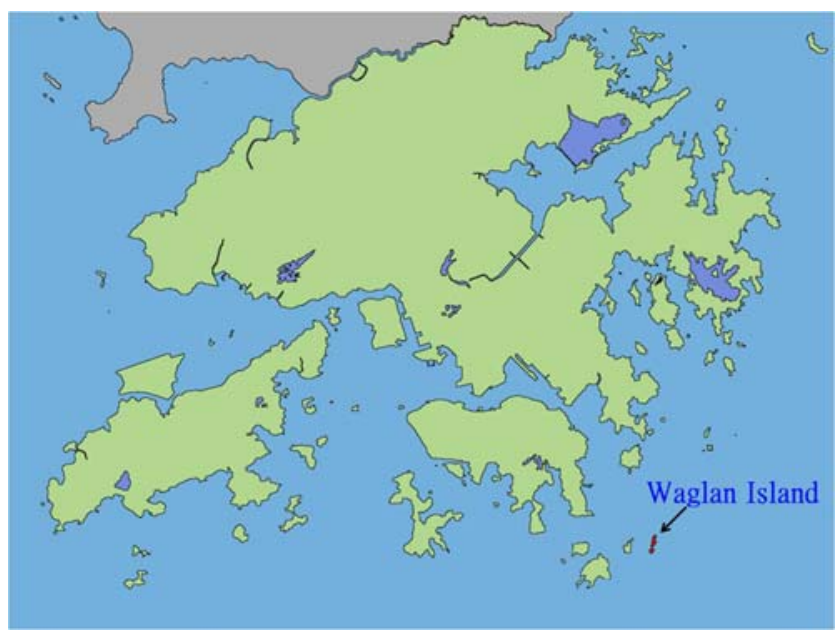

Figure 1. Location of Waglan Island in Hong Kong 


\subsection{Typhoon Wind Data}

Typhoon wind field data for the Western North Pacific region are recorded every six hours by the HKO. The typhoon wind field data from 1947 to 2006 are available to the authors, but only typhoons of their tracks within a radius of $250 \mathrm{~km}$ from the Waglan Island are considered in this study.

\subsection{Probability Distributions of Key Parameters}

The central pressure difference $\Delta p_{0}$ is the difference between the central pressure and the ambient pressure at zero plane. It plays a very important role in estimating design typhoon wind speeds. Both lognormal distribution and 3-parameter Weibull distribution are used to fit the observed data. The observed and fitted probability distribution function (PDF) and cumulative distribution function (CDF) of the central pressure difference $\Delta p_{0}$ are shown in Figure 2, and the results of statistical parameter estimation are listed in Table 1 , in which $\mu_{\ln }$ and $\sigma_{\ln }$ are the mean value and standard deviation of the lognormal distribution; and $\gamma, \beta, \eta$ are the location parameter, shape parameter and scale parameter of the 3-parameter Weibull distribution.

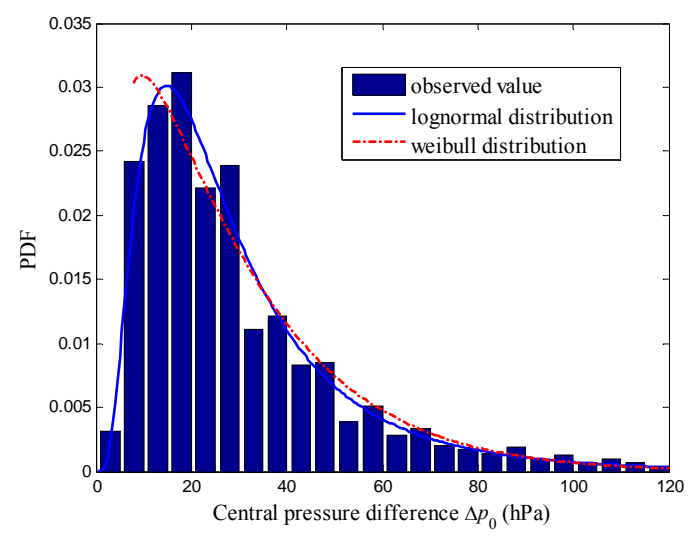

(a) PDF

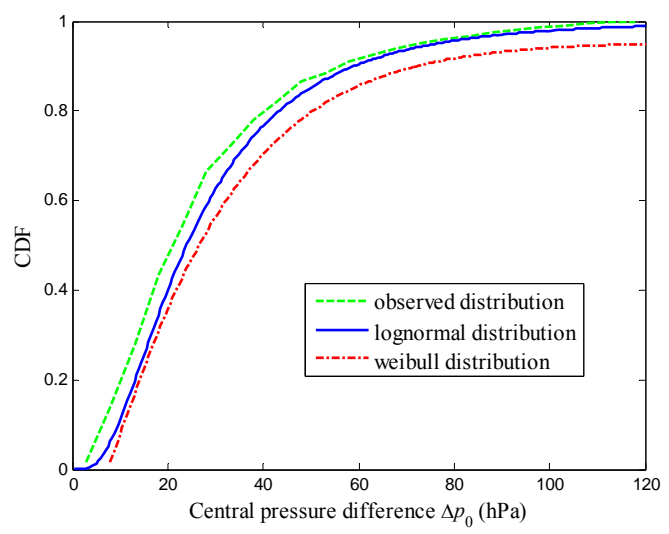

(b) $\mathrm{CDF}$

Figure 2. Observed and Fitted Distributions of Central Pressure Difference $\Delta p_{0}$

Table 1. Key Parameters and Probability Distributions of Typhoons

\begin{tabular}{|c|c|c|c|c|c|}
\hline Parameter & Distribution & Parameters in distribution & $D$ & $d_{F, n}$ & $K-S$ test \\
\hline \multirow{2}{*}{$\begin{array}{c}\text { translation velocity } \\
c(\mathrm{~m} / \mathrm{s})\end{array}$} & Normal & $\mu=19.1 \sigma=9.04$ & 0.065 & \multirow{2}{*}{0.019} & \multirow{2}{*}{ Lognorma } \\
\hline & Lognormal & $\mu_{\ln }=2.83 \sigma_{\ln }=0.53$ & 0.027 & & \\
\hline \multirow{2}{*}{$\begin{array}{c}\text { approach angle } \\
\beta \text { (degree) }\end{array}$} & Normal & $\mu=-46.4 \sigma=58.9$ & 0.194 & \multirow[b]{2}{*}{0.029} & \multirow[b]{2}{*}{ bi-normal } \\
\hline & bi-normal & $\begin{array}{c}\mu_{1}=-69.2 \sigma_{1}=26.4 a_{1}=0.828 \\
\mu_{2}=-69.2 \sigma_{2}=26.4\end{array}$ & 0.027 & & \\
\hline \multirow{2}{*}{$\begin{array}{c}\text { central pressure } \\
\text { difference } \\
\Delta p_{0}(\mathrm{hPa})\end{array}$} & Lognormal & $\mu_{\ln }=-3.19 \sigma_{\ln }=0.70$ & 0.035 & \multirow{2}{*}{0.026} & \multirow{2}{*}{ Lognormal } \\
\hline & Weibull & $\gamma=6.07 \beta=1.12 \eta=25.56$ & 0.195 & & \\
\hline $\begin{array}{c}\text { radius to maximum } \\
\text { wind } \\
r_{\mathrm{m}}(\mathrm{km})\end{array}$ & Lognormal & $\mu_{\mathrm{ln}}=4.19 \sigma_{\mathrm{ln}}=1.01$ & 0.027 & 0.031 & Lognormal \\
\hline \multirow{2}{*}{$\begin{array}{l}\text { minimum of closest } \\
\text { distance } d_{\min }(\mathrm{km})\end{array}$} & Uniform & $v=0.002$ & 0.046 & \multirow{2}{*}{0.027} & \multirow{2}{*}{ trapezoidal } \\
\hline & Trapezoidal & $A=0.0017 B=0.0023$ & 0.021 & & \\
\hline $\begin{array}{c}\text { annual occurrence rate } \\
\lambda \\
\end{array}$ & Poisson & $\lambda=2.9$ & & & \\
\hline
\end{tabular}


The translation velocity $c$ of a typhoon is determined by using the distance of the typhoon center between the two recorded points divided by 6-hours from the typhoon wind field data base. The results show that the translation velocity ranges from $2 \mathrm{~km} / \mathrm{h}$ to $65 \mathrm{~km} / \mathrm{h}$. Both normal distribution and lognormal distribution are used to fit the observed data. The observed and fitted PDF and CDF of the translation velocity $c$ are shown in Figure 3, and the results of statistical parameter estimation are listed in Table 1, in which $\mu$ and $\sigma$ are the mean value and standard deviation of the normal distribution.

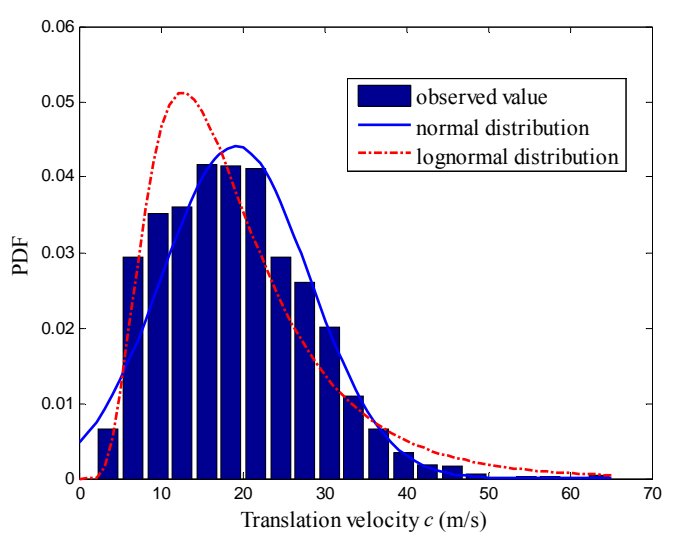

(a) PDF

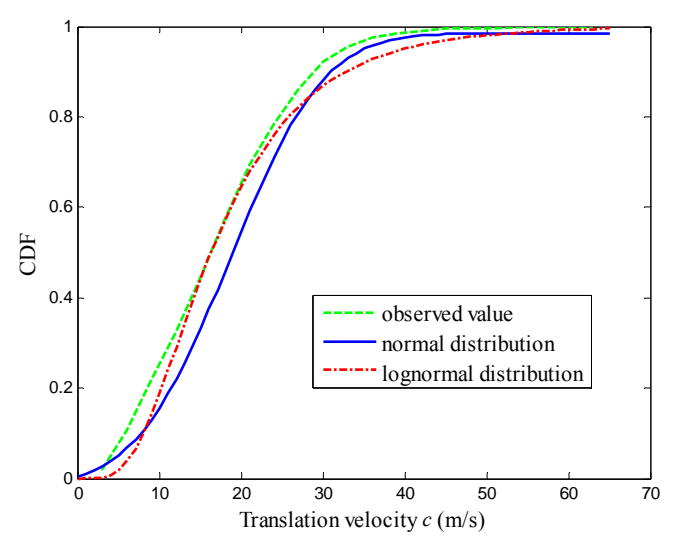

(b) $\mathrm{CDF}$

Figure 3. Observed and Fitted Distributions of Translation Velocity $c$

The approach angle $\theta$ of a typhoon indicates the typhoon moving direction and it is also estimated based on the adjacent positions of the typhoon center in a 6-hour interval. It is expressed to the nearest 10 degree with respect to the north clockwise. The observed data are fitted using both normal distribution and bi-normal distribution. The observed and fitted PDF and CDF of the approach angle $\theta$ are shown in Figure 4, and the results of statistical parameter estimation are listed in Table 1, in which $\mu_{1}$ and $\mu_{2}, \sigma_{1}$ and $\sigma_{2}$ are the mean values and standard deviations of the two random variables in the bi-normal distribution, and $a_{1}$ is the weighting factor.

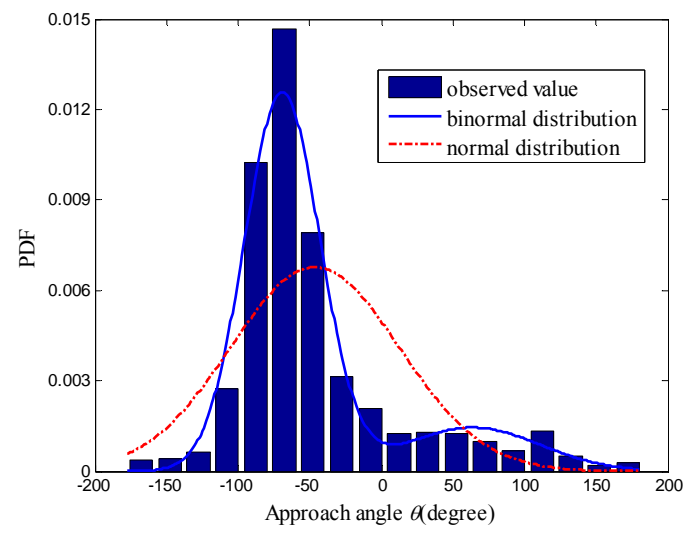

(a) PDF

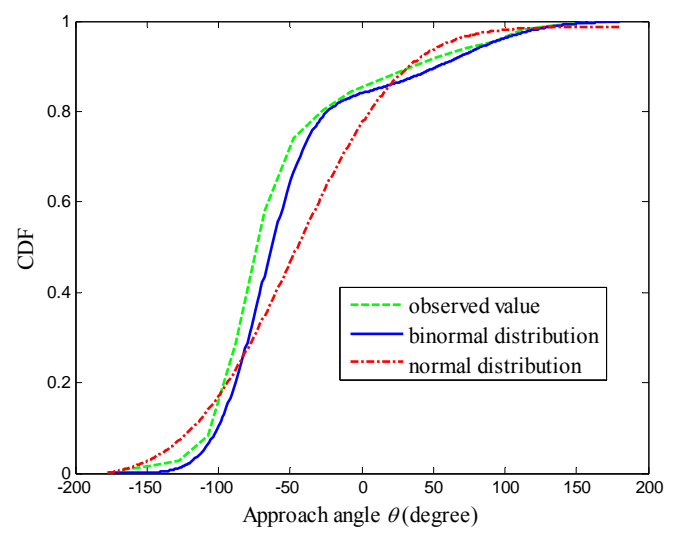

(b) $\mathrm{CDF}$

Figure 4. Observed and Fitted Distributions of Approach Angle $\theta$

The minimum of closest distance $d_{\min }$ is the minimum perpendicular distance from the simulation point to the typhoon translation direction and can be estimated according to the positions of both typhoon center and location of interest as well as the moving direction of the typhoon. It is defined 
as positive if the concerned location is located to the right of typhoon moving direction, otherwise negative. The observed minimum of closet distance is fitted by using uniform distribution and trapezoidal distribution. The observed and fitted PDF and CDF of the minimum of closest distance $d_{\min }$ are shown in Figure 5, and the results of statistical parameter estimation are listed in Table 1, in which $v$ is the value in the uniform distribution; $A$ and $B$ are the minimum and maximum values of trapezoidal probability density function.

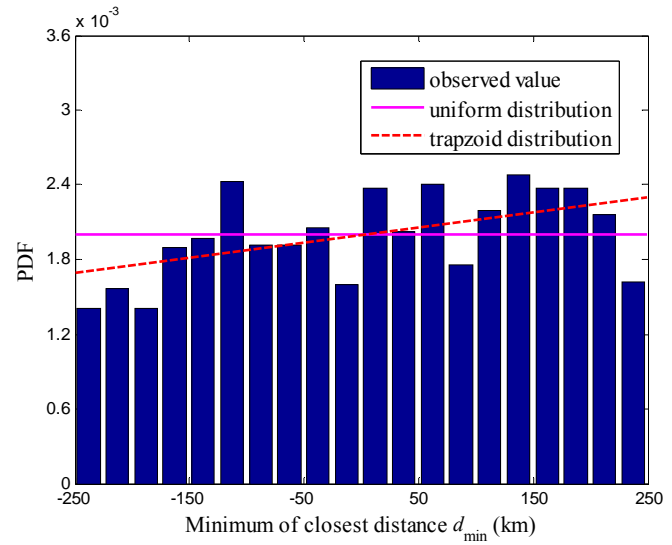

(a) PDF

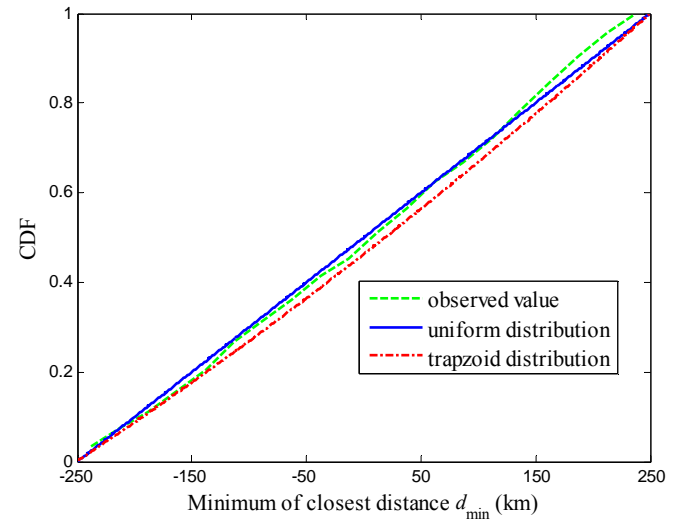

(b) $\mathrm{CDF}$

Figure 5. Observed and Fitted Distributions of Minimum of Closest Distance $d_{\min }$

The radius to maximum wind $r_{\mathrm{m}}$ describes the range of most intensive typhoon wind speed. It can be estimated according to the method proposed by Anthes [22]. The observed data are fitted by using the lognormal distribution only. The observed and fitted PDF and CDF of the radius to maximum winds $r_{\mathrm{m}}$ are shown in Figure 6, and the results of statistical parameter estimation are listed in Table 1.

The annual occurrence rate $\lambda$ means the typhoon occurrence times of typhoon per year and it is modeled by using the Poisson distribution only. The result is also shown in Table 1.

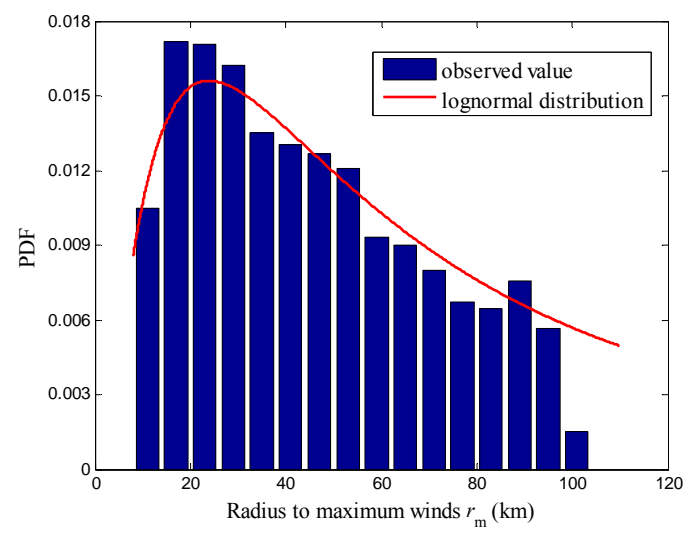

(a) PDF

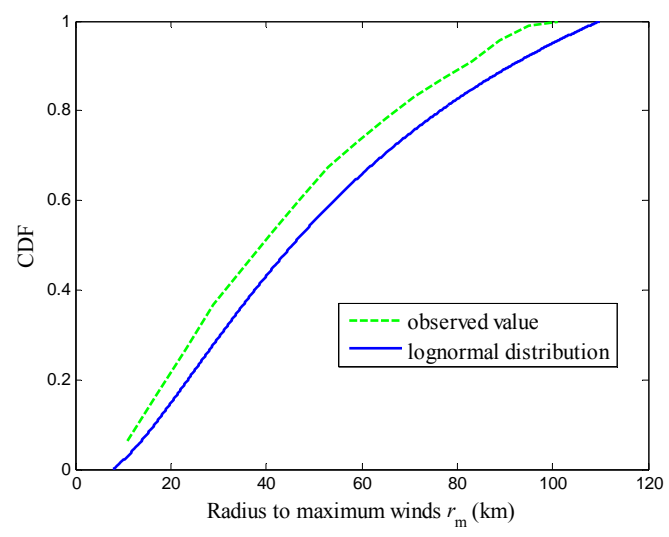

(b) $\mathrm{CDF}$

Figure 6. Observed and Fitted Distributions of Radius to Maximum Wind $r_{\mathrm{m}}$ 


\subsection{K-S Test}

Since the $K-S$ test can avoid measurement bias and can be effectively applied to 5 , but preferably 10 or more observations, the $K-S$ test is performed here to determine the distribution by providing the best fit to the historical typhoon wind field data except for the annual occurrence rate $\lambda$.

The $K-S$ test is based upon the maximum distance $D$ between the probability cumulative distribution function $F(x)$ and the empirical cumulative distribution function $F_{n}(x)$.

$$
D=\max \left|F(x)-F_{n}(x)\right|
$$

The distribution function $F(x)$ is rejected if $D$ is much greater than the specified value $d_{F, n}$. By choosing the cutting off upper-tail area of 0.05 , the value of $d_{F, n}$ is defined as:

$d_{F, n}=\frac{1.36}{\sqrt{n}}$

where $n$ is the number of observations.

Based on the $K-S$ test, the distributions of key parameters discussed in Section 3.2 are finally decided and shown in Table 1 together with their respective $D$ values.

\subsection{Typhoon Wind Decay Model Parameters}

The parameters in the typhoon wind decay model can also be obtained through the linear regression analysis of the typhoon wind field data selected. The results from the regression analysis indicate that the two constants $a_{0}$ and $a_{1}$ are 0.0237 and 0.00012 , respectively. The mean value and standard deviation are 0.0 and 0.0193 for the random variable $\varepsilon$ and -0.011 and 0.162 for the random variable $b$. Figure 7 shows the relationship between the decay constant $a$ and $\Delta p_{0 \mathrm{n}}$ at landfall. It is noted that the correlations between the typhoon parameters above are not considered in this study. How to generate typhoons with the correlations between typhoon parameters deserves further investigation.

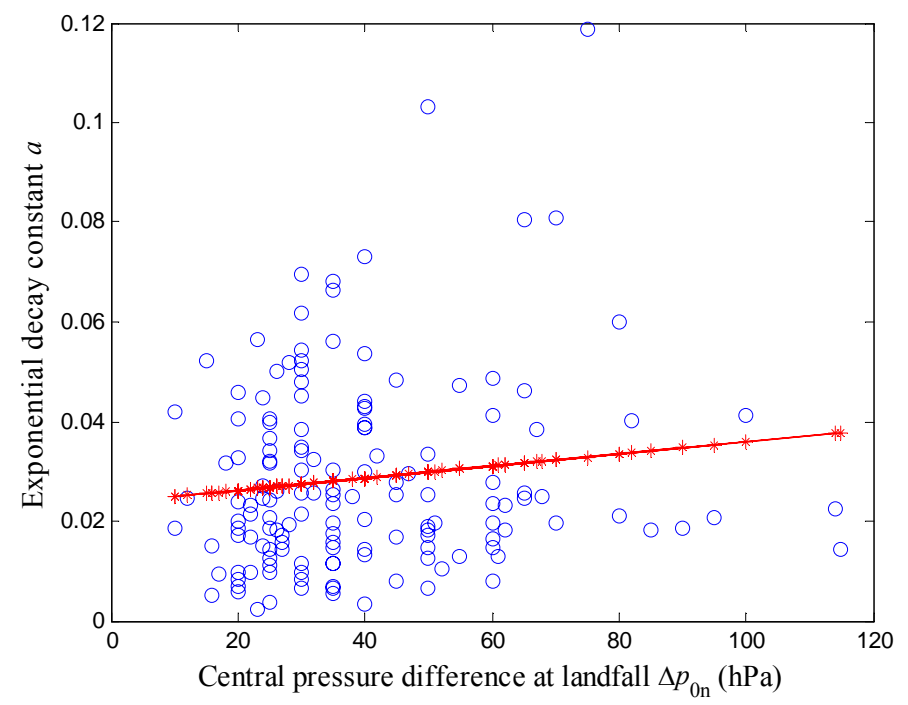

Figure 7. Relationship between Decay Constant $a$ and $\Delta p_{0 \mathrm{n}}$ at Landfill 


\subsection{Procedure for Estimating Extreme Wind Speeds and Averaged Wind Profiles}

The basic steps of using the Monte Carlo simulation method to estimate extreme wind speeds for the area concerned can be summarized as follows: (a) generate a set of typhoon key parameters based on the established probability distributions; (b) generate a typhoon wind field at its initial position based on the generated typhoon key parameters and then let the typhoon move using the refined typhoon wind field model, in which the central pressure difference $\Delta p_{0}$ is held constant until landfall and afterwards the central pressure difference is determined by the typhoon wind decay model; (c) computer the mean wind speed and direction at the area concerned due to the moving typhoon; (d) repeat above steps for thousands of typhoons; and (e) carry out the extreme wind analysis to determine design typhoon wind speeds in terms of a given return period. In the simulation process of the study, the typhoon translation velocity $c$ is held constant for each typhoon. Each simulated typhoon travels along a straight line path. Furthermore, to simulate the typhoon wind field at an hourly interval, all the parameters are linearly interpolated from the available 6-hourly track information provided by HKO.

The steps (a) to (d) of using the Monte Carlo simulation method to estimate extreme wind speeds provide not only thousands of hourly mean wind speeds and directions but also thousands of mean wind speed profiles at the Waglan Island. Since typhoon mean wind speed profiles vary with the distance from the typhoon center, the obtained thousands of mean wind speed profiles shall be classified according to the distance from the typhoon center. In this study, only the two regions, eye-wall region and outer-vortex region, are considered. All the mean wind speed profiles belonging to either the region are averaged to obtain the averaged typhoon wind profiles for the eye-wall region and the outer-vortex region. These averaged typhoon wind profiles are also called the design typhoon wind profiles.

\section{EXTREME WIND SPEED ANALYSIS}

\subsection{Basic Theory of Extreme Wind Speed Analysis}

The basic theory of extreme wind speed analysis based on the Monte Carlo simulation method can be found in Simiu and Scanlan [23]. Denote $F_{v}$ as the probability that the wind speed in any one typhoon simulated is less than some value $v$. The probability that the highest wind $U$ in $n$ typhoons simulated is less than $v$ can be written as

$F(U<v \mid n)=\left(F_{v}\right)^{n}=F_{v}^{n}$

According to the total probability theorem, the probability that $U<v$ in $\tau$ years is:

$F(U<v, \tau)=\sum_{n=0}^{\infty} F(U<v \mid n) p(n, \tau)$

where $p(n, \tau)$ is the probability that $n$ typhoons will occur in $\tau$ years.

Assuming that $p(\mathrm{n}, \tau)$ follows a Poisson distribution, Eq. 9 becomes

$F(U<v, \tau)=\sum_{n=0}^{\infty} F_{v}^{n} \frac{(\lambda \tau)^{n} e^{-\lambda \tau}}{n !}=e^{-\lambda \tau} \sum_{n=0}^{\infty} \frac{\left(\lambda \tau F_{v}\right)^{n}}{n !}=e^{-\lambda \tau\left(1-F_{v}\right)}$ 
where $\lambda$ is the annual occurrence rate of typhoons in the area of interest for the site being considered. When $\tau=1, F(U<v, \tau)$ becomes the probability of occurrence of wind speeds less than $v$ in any one year.

$F(U<v)=\sum_{n=0}^{\infty} F_{v}^{n} \frac{(\lambda)^{n} e^{-\lambda}}{n !}=e^{-\lambda} \sum_{n=0}^{\infty} \frac{\left(\lambda F_{v}\right)^{n}}{n !}=e^{-\lambda\left(1-F_{v}\right)}$

Suppose that $m$ typhoons are simulated using the Monte Carlo simulation method and the maximum wind speed induced by each typhoon for the site can be arranged in an ascending order as $v_{1}, v_{2}, \ldots v_{m}$. By considering the wind speed $v_{i}$, the probability that $U<v_{i}$ in any one typhoon can be determined by

$F_{v_{i}}=\frac{i}{m+1}$

The probability of occurrence of wind speeds less than $v_{i}$ in any one year is

$F\left(U<v_{i}\right)=e^{-\lambda\left(1-\frac{i}{m+1}\right)}$

Extreme wind speeds in typhoons are usually fitted with one of the family of the generalized Extreme Value Distributions (GEV). The Type I Extreme Value (Gumbel) Distribution is used in the Hong Kong wind code. The Gumbel distribution function can be written in the form:

$F(v)=\exp [-\exp (-\alpha(v-\mu))]$

where $v$ is the annual maximum wind speed; $\alpha$ is the dispersion; and $\mu$ is the mode. By using Eq. 13, the dispersion and the mode in Eq. 14 can be estimated.

The design wind speed for a given return period $T_{R}$ (in year) and a given structure life $N$ (in year) can be finally determined by

$[F(v)]^{N}=\left[1-\frac{1}{T_{R}}\right]^{N}=1-R$

where $R$ is the associated risk of having a wind speed higher than $v$ in $N$ years.

\subsection{Extreme Wind Speed Analysis Using the Refined Typhoon Wind Field Model}

The Waglan Island meteorological station in Hong Kong is chosen as a reference location for extreme wind speed analysis. The surrounding condition around the station can be regarded as an open terrain, and the adjusted measurement height of the station is $90 \mathrm{~m}$ above the sea level. By using the Monte Carlo simulation method, nearly 10,000 typhoons are generated and the hourly mean wind speed and direction of each typhoon at the reference height of $90 \mathrm{~m}$ of the station are calculated. The Gumbel distribution is employed for extreme wind speed value analysis. Both non-directional and directional design wind speeds of 50-year return period at the Waglan Island anemometer height are computed. The results show that the non-directional design wind speed of a 50 year return period at the station is $48.2 \mathrm{~m} / \mathrm{s}$. The directional design wind speeds of a 50 year return period in 36 direction sectors at the Waglan Island obtained from the refined typhoon wind 
field model are shown in Figure 8. It can be seen that the asymmetrical directional distribution of design wind speeds can be produced satisfactorily by using the refined typhoon wind field model.

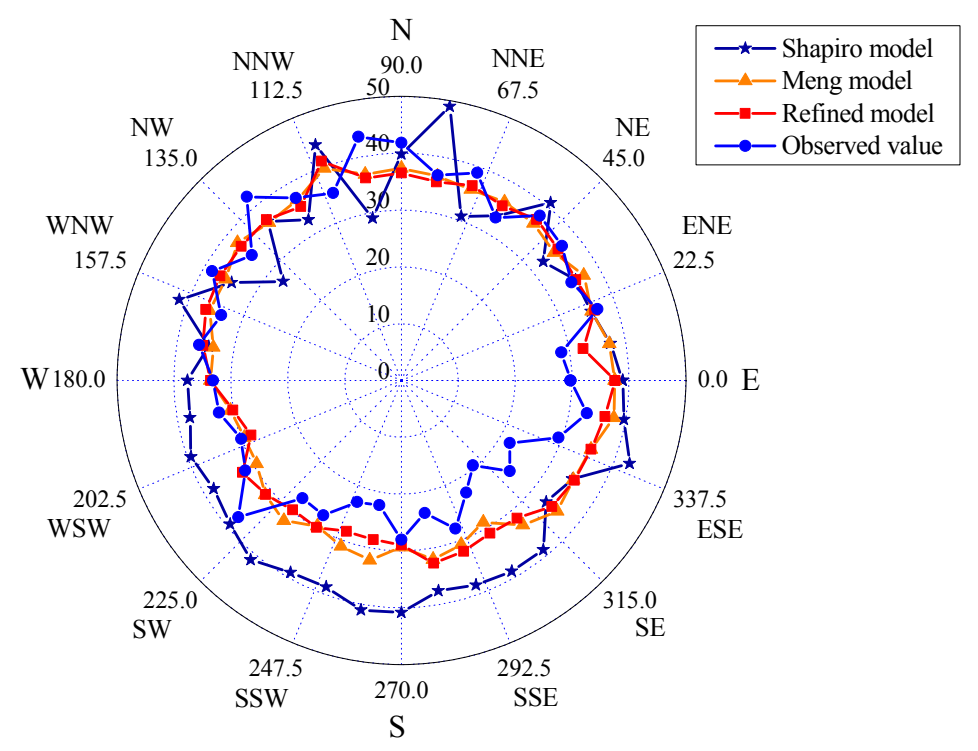

Figure 8. Directional Design Wind Speeds of a 50 Year Return Period at Waglan Island

\subsection{Extreme Wind Speed Analysis Based on Wind Measurement Data}

Hourly mean wind speeds and directions at the Waglan Island are recorded by HKO. The wind measurement data during a period from 1970 to1999 are available to the authors. The data include the hourly mean wind speed and mean wind direction. The data are allocated according to wind direction at a 10 degree interval. The annual maximum wind speed in each of 36 direction sectors and the annual maximum wind speed among all the directions are obtained for each year, from which extreme wind speed analysis is performed. By using the Gumbel distribution, the non-directional design wind speed of a 50 year return period at the Waglan Island is $46.9 \mathrm{~m} / \mathrm{s}$, which is also provided by the Hong Kong wind code. The directional design wind speeds of a 50 year return period in each direction at the Waglan Island obtained from the wind measurement data are shown in Figure 8. It is noted that in Figure 8, northern wind refers to wind blowing from the North.

\subsection{Extreme Wind Speed Analysis Using the Meng Model and the Shapiro Model}

By using the Monte Carlo simulation method together with the Meng model or the Shapiro model, nearly 10,000 typhoons are generated and the hourly mean wind speed and direction for each typhoon at the reference height of $90 \mathrm{~m}$ of the station are calculated. The Gumbel distribution is also employed for extreme wind speed value analysis. Both non-directional and directional design wind speeds of 50-year return period at the Waglan Island anemometer height are computed by using the Meng model or the Shapiro model. The results show that the non-directional design wind speed of a 50 year return period at the station is $50.7 \mathrm{~m} / \mathrm{s}$ from the Meng model and $54.3 \mathrm{~m} / \mathrm{s}$ from the Shapiro model. The directional design wind speeds of a 50 year return period in 36 direction sectors at the Waglan Island obtained from the Meng model or the Shapiro model are also shown in Figure 8. 


\subsection{Comparison of Results and Discussion}

The design wind speed of a 50 year return period predicted by the refined typhoon wind field model for the Waglan Island is $48.2 \mathrm{~m} / \mathrm{s}$. This value is slightly higher than the design wind speed of $46.9 \mathrm{~m} / \mathrm{s}$ predicted based on the wind measurement data. The error between the refined typhoon wind field model and the wind measurement data may be because the number of typhoons used in the Monte Carlo simulation method is much larger than that actually recorded in the Waglan Island during the concerned period. On the other hand, the design wind speeds of a 50 year return period predicted by the Shapiro model and the Meng model are, respectively, $54.3 \mathrm{~m} / \mathrm{s}$ and $50.7 \mathrm{~m} / \mathrm{s}$.

In considering the effects of wind direction on design wind speeds, the largest design wind speeds of a 50 year return period predicted based on the wind measurement data and by using the refined typhoon wind field model are $43.6 \mathrm{~m} / \mathrm{s}$ and $41.1 \mathrm{~m} / \mathrm{s}$ and occur in the direction of 90 degree and 110 degree, respectively. The largest design wind speeds of a 50 year return period predicted by using the Meng model and Shapiro model are $39.7 \mathrm{~m} / \mathrm{s}$ and $49.0 \mathrm{~m} / \mathrm{s}$ and occur in the direction of 110 degree and 80 degree, respectively. The smallest design wind speeds of a 50 year return period predicted based on the wind measurement data and by using the refined typhoon wind field model are $19.5 \mathrm{~m} / \mathrm{s}$ and $28.2 \mathrm{~m} / \mathrm{s}$ and happen in the direction of 300 degree and 200 degree or 250 degree, respectively. However, the smallest design wind speeds of a 50 year return period predicted by using the Meng model and the Shapiro model are $28.8 \mathrm{~m} / \mathrm{s}$ and $27.1 \mathrm{~m} / \mathrm{s}$ and happen in the direction of 300 degree and 140 degree, respectively. The simulation errors between the refined typhoon wind field model, the Shapiro model, the Meng model and the wind measurement data are shown in Figure 9 for 36 direction sectors. The mean errors produced by the refined typhoon wind field model, the Meng model, and the Shapiro model are $4.14 \mathrm{~m} / \mathrm{s}, 4.64 \mathrm{~m} / \mathrm{s}$, and $8.49 \mathrm{~m} / \mathrm{s}$, respectively. The standard deviations of the errors produced by the refined typhoon wind field model, the Meng model, and the Shapiro model are $3.37 \mathrm{~m} / \mathrm{s}, 3.54 \mathrm{~m} / \mathrm{s}$, and $5.24 \mathrm{~m} / \mathrm{s}$, respectively. Although the results from the refined typhoon wind field model and the Meng model are similar, it will be found in the following that the two models give similar results only in the lower positions above the ground. The two models depart from each other in high positions above the ground.

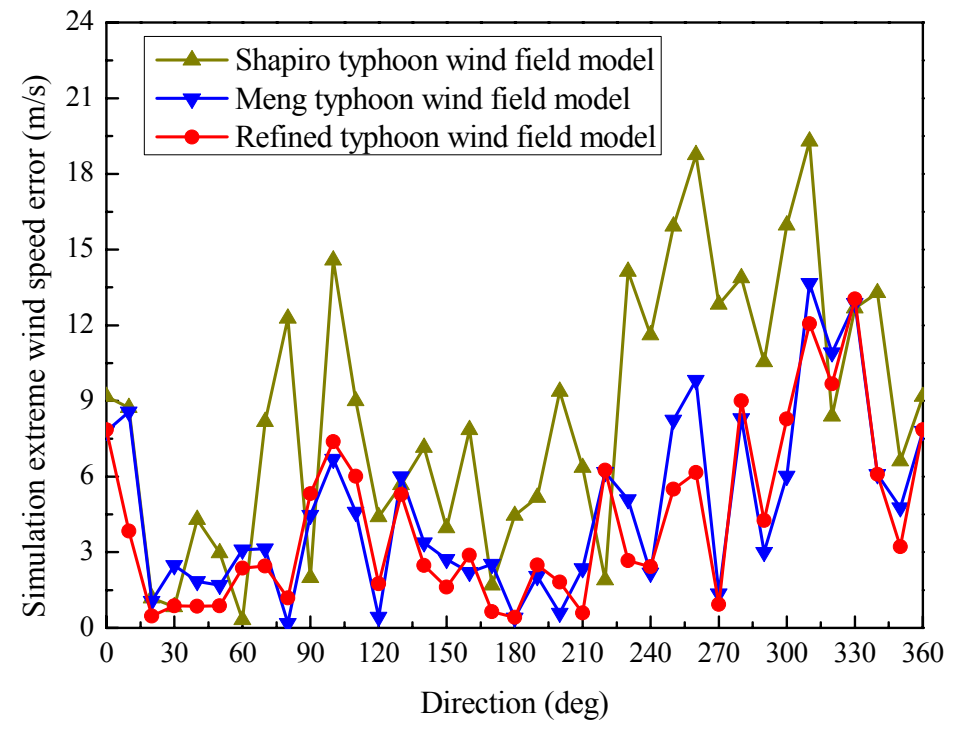

Figure 9. Simulation Errors Among the Three Models and Measurement Data 


\section{MEAN WIND SPEED PROFILE ANALYSIS}

One of the major factors affecting the magnitude of wind loading on buildings and structures is the mean wind speed profile. By using the Monte Carlo simulation method described in Section 3.5, hourly mean wind speed profiles from thousands of typhoons are generated by using the refined typhoon wind field model and the Meng model. Only the mean wind speed profiles with the mean wind speed over $5 \mathrm{~m} / \mathrm{s}$ at $10 \mathrm{~m}$ height and the distance from the typhoon center within the range from $r_{\mathrm{m}}$ to $5 r_{\mathrm{m}}$ are counted and analyzed.

The development of the global positioning system (GPS)-based drop sondes (Hock and Franklin [24]) has made it possible to obtain mean wind speed profiles within nearly all positions of a typhoon. By using the wind profiles obtained by GPS drop sondes at Atlantic, Eastern and Central Pacific during years of 1997-1999, Powell et al. [25] and Franklin et al. [26] obtained averaged eye-wall wind profiles and outer-vortex wind profiles. By averaged all the mean wind speed profiles within the two regions generated by the refined typhoon wind field model and the Meng model, the averaged eye-wall and outer-vortex wind profiles are obtained and compared with these measured wind profiles.

Figure 10 displays the averaged wind profiles predicted by the refined typhoon wind field model and the Meng model for the eye-wall region (from $r_{\mathrm{m}}$ to $2 r_{\mathrm{m}}$ ) and those obtained by Powell et al. [25] and Franklin et al. [26] from the measured data. It can be seen that the averaged wind profile predicted by the refined typhoon wind field model is close to that by the Meng model only in the lower positions (less than $300 \mathrm{~m}$ above the ground). With increasing height greater than $300 \mathrm{~m}$, the averaged wind profiles given by the two models depart from each other. The averaged wind profile predicted by the refined typhoon wind field model is more close to the measured ones than that by the Meng model. This is because the variation of central pressure difference with height is taken into consideration in the refined typhoon wind field model rather than the Meng model. The relative difference of the averaged wind profile from the refined typhoon wind field model to that given by Powell et al. [25] is $6 \%$ at $700 \mathrm{~m}$ high. The relative difference of the averaged wind profile from the Meng model to that given by Powell et al. [25] is $16 \%$ at $700 \mathrm{~m}$ high.

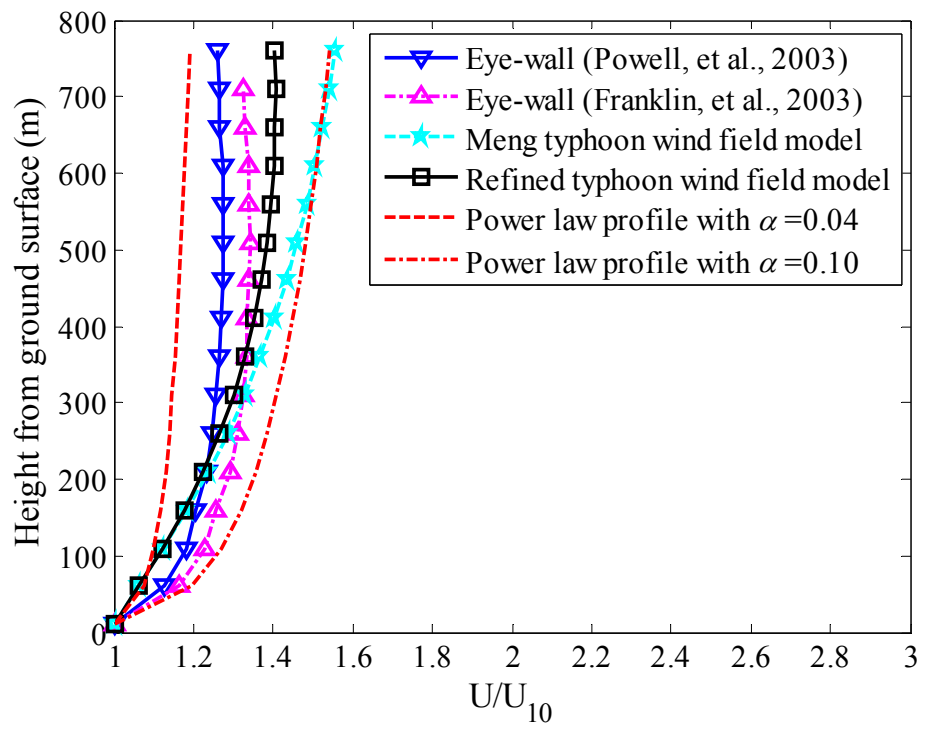

Figure 10. Comparison of Wind Speed Profiles in Eye-wall Region 
Figure 11 displays the averaged wind profiles predicted by the refined typhoon wind field model and the Meng model for the outer-vortex region (from $2 r_{\mathrm{m}}$ to $5 r_{\mathrm{m}}$ ) and that obtained by Franklin et al. [26] from the measured data. In lower positions (less than $300 \mathrm{~m}$ above the ground), the averaged wind profiles predicted by the refined typhoon wind field model and Meng model are similar but the wind speeds are relatively smaller compared with the measured ones given by Franklin et al. [26]. In higher positions (greater than $300 \mathrm{~m}$ above the ground), the refined typhoon wind field model gives a good estimation on wind speeds compared with the measured mean wind profile, but the Meng model overestimates wind speeds compared with the measured mean wind profile. The relative difference of the averaged wind profile from the refined typhoon wind field model to the measured mean wind profile is $1 \%$ at $700 \mathrm{~m}$ high. The relative difference of the averaged wind profile from the Meng model to the measured mean wind profile is $8 \%$ at $700 \mathrm{~m}$ high.

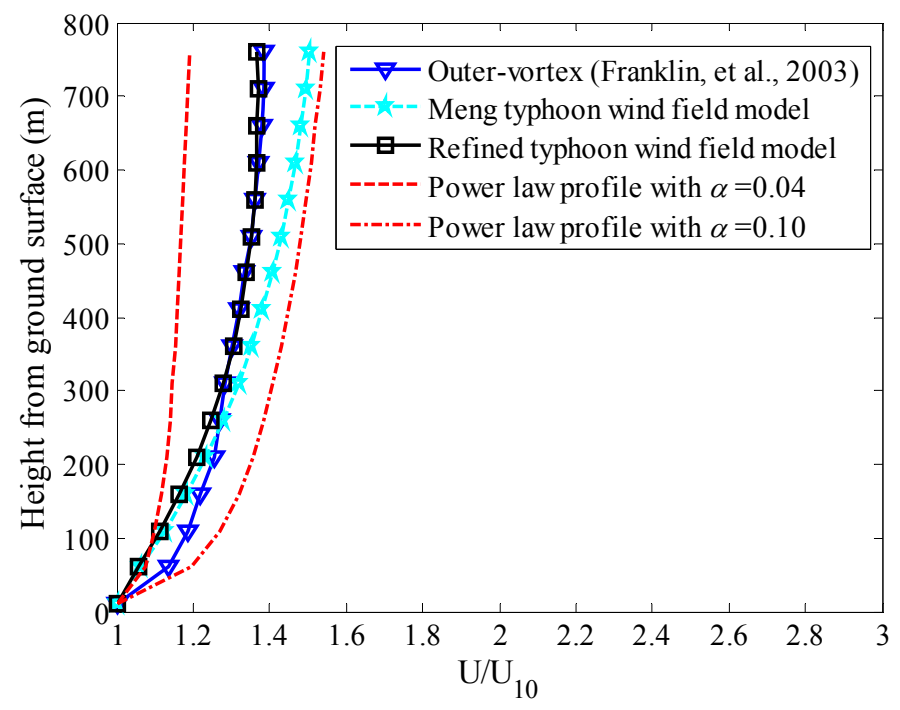

Figure 11. Comparison of Wind Speed Profiles in Outer-vortex Region

\section{CONCLUSIONS}

The Monte Carlo simulation method has been used in conjunction with the refined typhoon wind field model and the typhoon wind decay model to predict the non-directional and directional design typhoon wind speeds at the Waglan Island of Hong Kong. The design wind speed of a 50 year return period predicted by the refined typhoon wind field model is $48.2 \mathrm{~m} / \mathrm{s}$ among all the directions. In consideration of effects of wind direction, the largest and smallest design wind speeds of a 50 year return period predicted by using the refined typhoon wind field model are $41.1 \mathrm{~m} / \mathrm{s}$ and 28.2 $\mathrm{m} / \mathrm{s}$, respectively. The same approach, but using the Meng model and the Shapiro model, has also been applied to predict the non-directional and directional design typhoon wind speeds at the Waglan Island and compare with the results obtained based on direct measurement data. The analysis of prediction errors show that the average error produced by the refined typhoon wind field model is the smallest compared with these produced by the Meng model and the Shapiro model. The averaged wind profiles at the Waglan Island in both the eye-wall and outer-vortex regions have been also predicted by the refined typhoon wind field model and the Meng model and compared with the field measurement data from GPS-based drop snodes. The results show that the refined typhoon wind field model could depict typhoon wind speed profiles in both the eye-wall region and the outer-vortex region and the predicted wind profiles are more close to the measured ones than the Meng model. This is because the refined typhoon wind field model includes the effect of temperature and the variation of central pressure difference with height. 
Although the results predicted by the refined typhoon wind field model are satisfactory, this study is preliminary for Hong Kong and further investigations are required on some important issues such as representative roughness length, correlation of typhoon parameters, and impact of climate change.

\section{ACKNOWLEDGEMENTS}

The work described in this paper was financially supported by the Harbin Institute of Technology Shenzhen Graduate School through a PhD studentship to the first author, and by The Hong Kong Polytechnic University through its Niche Area Program (PolyU 1-BB20 and 1-BB68) and the Natural Science Foundation of China through its Key Research Program (NSFC 50830203) to the second author. The support from the Hong Kong Observatory to allow the authors to access the measured data for academic purpose only is particularly appreciated. Any opinions and conclusions presented in this paper are entirely those of the authors.

\section{REFERENCES}

[1] Russell, L.R., "Probability Distributions for Texas Gulf Coast Hurricane Effects of Engineering Interest", PhD thesis, Stanford University, Stanford, California, 1968.

[2] Russell, L.R., "Probability Distributions for Hurricane Effects", Journal of the Waterways, Harbors and Coastal Engineering Division, 1971, Vol. 97, No. 1, pp. 139-154.

[3] Martin, G.S., "Probability Distributions for Hurricane Wind Gust Speeds on the Australian Coast", Proc. I.E. Aust. Conf. on Applied Probability Theory to Structural Design, Melbourne, 1974.

[4] Tryggvason, B.V., Davenport, A.G. and Surry, D., "Predicting Wind-induced Response in Hurricane Zones", Journal of the Structural Division, 1976, Vol. 102, No. 12, pp. 2333-2350.

[5] Batts, M.E., Simiu, E. and Russell, L.R., "Hurricane Wind Speeds in the United States", Journal of the Structural Division, 1980, Vol. 106, No. 10, pp. 2001-2016.

[6] Georgiou, P.N., Davenport, A.G. and Vickery, B.J., "Design Wind Speeds in Regions Dominated by Tropical Cyclones", Journal of Wind Engineering and Industrial Aerodynamics, 1983, Vol. 13, pp. 139-152.

[7] Fujii, T. and Mitsuta, Y., "Simulation of Winds in Typhoons by a Stochastic Model", Journal of Wind Engineering, 1986, Vol. 28, pp. 1-12.

[8] Vickery, P.J. and Twisdale, L.A., "Wind-field and Filling Models for Hurricane Wind-speed Predictions", Journal of Structural Engineering, 1995, Vol. 121, No. 11, pp. 1700-1709.

[9] Vickery, P.J., Skerlj, P.F. and Twisdale, L.A, "Simulation of Hurricane Risk in the U.S. Using Empirical Track Model”, Journal of Structural Engineering, 2000, Vol. 126, No. 10, pp. 1222-1237.

[10] Vickery, P.J. and Twisdale, L.A., "Prediction of Hurricane Wind Speeds in the United States", Journal of Structural Engineering, 1995, Vol. 121, No. 11, pp. 1691-1699.

[11] Chow, S.H., "A Study of the Wind Field in the Planetary Boundary Layer of a Moving Tropical Cyclone", MS thesis, School of Engineering and Science, New York University, New York, NY, 1971.

[12] Gomes, L. and Vickery, B.J., "On the Prediction of Tropical Cyclone Gust Speeds along the Northern Australian Coast”, Inst. Eng. Aust. C.E. Trans. CE18, 1976, Vol. 2, pp. 40-49.

[13] Holland, G.J., "An Analytical Model of the Wind and Pressure Profile in Hurricanes", Monthly Weather Review, 1980, Vol. 108, pp. 1212-1218.

[14] Shapiro, L.J., "The Asymmetric Boundary Layer Flow under a Translating Hurricane", Journal of the Atmospheric Sciences, 1983, Vol. 40, pp. 1984-1998. 
[15] Georgiou, P.N., "Design Wind Speeds in Tropical Cyclone-prone Regions", PhD Thesis, Department of Civil Engineering, University of Western Ontario, Canada, 1985.

[16] Thompson, E.F. and Cardone, V.J., "Practical Modeling of Hurricane Surface Wind Fields", Journal of Waterway, Port, Coastal, and Ocean Engineering, 1996, Vol. 122, No. 4, pp. 195-205.

[17] Meng, Y., Matsui, M. and Hibi, K., "An Analytical Model for Simulation of the Wind Field in a Typhoon Boundary Layer", Journal of Wind Engineering and Industrial Aerodynamics, 1995, Vol. 56, pp. 291-310.

[18] Jin, Y., Thompson, T., Wang, Shouping and Liu, Chi-Sann, "A Numerical Study of the Effect of Dissipative Heating on Tropical Cyclone Intensity", Weather and Forecasting, 2007, Vol. 22, pp. 950-966.

[19] Kepert, J.D., "Observed Boundary Layer Wind Structure and Balance in the Hurricane Core. Part I: Hurricane Georges", Journal of the Atmospheric Sciences, 2006, Vol. 63, No. 2, pp. 2169-2193.

[20] Huang, W.F. and Xu, Y.L., "A Refined Model for Typhoon Wind Field Simulation in Boundary Layer", Advances in Structural Engineering-An International Journal, 2011 (in print).

[21] Holton, J.R., "An Introduction to Dynamic Meteorology", Fourth Edition, Academic Press, 2004.

[22] Anthes, R.A., "Tropical Cyclones: Their Evolution, Structure, and Effects", American Meteorological Society, Boston, 1982.

[23] Simiu, E. and Scanlan, R.H., "Wind Effects on Structures", Third Edition, John Wiley \& Sons, 1996.

[24] Hock, T.F. and Franklin, J.L., "The NCAR GPS Dropwindsonde", Bulletin of the American Meteorological Society, 1999, Vol. 80, No. 3, pp. 407-420.

[25] Powell, M.D., Vickery, P.J. and Reinhold T.A., "Reduced Drag Coefficient for High Wind Speeds in Tropical Cyclones", Nature, 2003, Vol. 422, pp. 279-283.

[26] Franklin, J.L., Black, M.L. and Valde, K., "GPS Dropwindsonde Wind Profiles in Hurricanes and Their Operational Implications", Weather and Forecasting, 2003, Vol. 18, pp. 32-44. 